\title{
RESILIENCIA Y TURISMO: EL CASO DE LA CIUDAD DE BAÑOS DE AGUA SANTA - ECUADOR
}

\author{
G. HERRERA ${ }^{1 *}$, G. RODRÍGUEZ ${ }^{2}$ \\ ${ }^{1}$ Universidad de las Fuerzas Armadas - ESPE Ecuador \\ ${ }^{2}$ Universidad Santiago de Compostela - España \\ gpherrera@espe.edu.ec*
}

Artículo recibido en marzo/2016 y aceptado en abril/2016

DOI: $10.15628 /$ holos.2016.4303

\section{RESUMEN}

En 1999, la ciudad turística de Baños de Agua Santa en Ecuador fue evacuada debido a la inminente erupción del volcán Tungurahua, el $90 \%$ de este territorio se encuentra en una zona de alto riesgo sísmico y volcánico, la evacuación destruiría la principal actividad económica que es el turismo, los habitantes en contra de las disposiciones gubernamentales deciden retornar y se ven en la necesidad de adaptarse a las nuevas condiciones que les ha obligado un proceso eruptivo que lleva más de
15 años, durante este tiempo la ciudad desarrolla procesos adaptativos que en este trabajo se lo explican desde el paradigma de la resiliencia y el modelo heurístico de panarquía siendo éste el principal objetivo. El modelo heurístico de panarquía explica a través de sus diferentes ciclos, los procesos de destrucción creativa e innovación que los evidencia el caso presentado; el principal hecho que se destaca es la evolución de la actividad turística en un nuevo ciclo panárquico.

PALAVRAS CLAVE: desastres naturales, turismo, impacto económico

\section{RESILIENCE AND TOURISM: THE CASE OF BAÑOS DE AGUA SANTA CITY - ECUADOR}

\begin{abstract}
In 1999, the touristic city of Baños de Agua Santa in Ecuador was evacuated due to imminent eruption of the Tungurahua volcano, $90 \%$ of this territory is in an area of high seismic and volcanic risk. The evacuation destroyed its main economic activity that is tourism. The inhabitants, in spite of government regulations decide to come back and adapt to the new conditions. During 15 years the volcano has been erupting and the city present
\end{abstract}

various adaptive processes. The main objective of this paper is explaining through the paradigm of resilience and heuristic model of panarchy these behaviors. The heuristic model panarchy explains the creative destruction processes and innovation that this case presented evidence; the main fact that stands out is the evolution of tourism activity across of new panarchycal cycle.

KEYWORDS: natural disaters, tourism, economic impact. 


\section{INTRODUCCÍON}

La ciudad de Baños de Agua Santa (1.815 msnm) se encuentra asentada sobre una meseta basáltica en las faldas del volcán Tungurahua (5.023 msnm) - Ecuador a $180 \mathrm{~km}$ de Quito y $35 \mathrm{~km}$ de Ambato. De acuerdo a la proyección censal, al 2015 la población es de 22.838 habitantes (población urbano y rual del municipio), cuenta con 3.150 empresas (INEC, 2013) y es reconocido como uno de los destinos turísticos más visitados de Ecuador. Esta realidad se contrasta con la vivida en 1999 cuando el Tungurahua presentara evidencias de una erupción inminente, por lo que las autoridades declararían la "alerta naranja" y promoverían una evacuación forzosa el 16 de octubre de ese año, dejando como resultado la destrucción de la estructura social y económica que obligó a la población a tomar medidas drásticas para retornar a su ciudad el 5 de enero de 2000, luego de graves enfrentamientos con la fuerza pública (Lane et al., 2003; Tobin \& Whiterford, 2002). Durante más de 15 años la ciudad se reinventó, aprendió a convivir con el desastre y encontró en éste oportunidades para el desarrollo llevándola a mejorar sus condiciones de vida, inclusive superando su realidad antes del desastre.

En busca de un marco teórico adecuado para comprender la complejidad de las variables que interactúan en la recuperación en zonas de desastre, este trabajo explica la dinámica de desarrollo de la ciudad de Baños entorno al impacto de la erupción del volcán Tungurahua sobre la base del modelo heurístico de panarquía, a la vez que identifica una serie de variables que se corresponden a un grupo de factores críticos, considerados pertinentes para la evaluación de la resiliencia comunitaria. Este estudio explica en las diferentes fases del modelo de panarquía el proceso de desarrollo de la ciudad de Baños antes y durante la erupción del Tungurahua, al ser el modelo una representación de los sistemas adaptativos complejos (Gunderson \& Holling, 2002), sus características, propiedades y dinámica se extrapolan a la población de estudio, obteniéndose un primer diagnósticos de sus variables de estado.

\section{PANARQUIA Y RESILIENCIA EN ZONAS DE DESASTRE}

Ante la necesidad de comprender el cambio adaptativo y la complejidad de las relaciones en los sistemas ecológicos, económicos y organizacionales, se adopta el término panarquía, cuyo objetivo fundamental es racionalizar la interacción entre el cambio y la persistencia, entre lo predecible y no predecible (Holling et al., 2002), es un modelo heurístico (Allen et al., 2014) que tiene la posibilidad de ajustarse a las ideas de jerarquías y escalas (García, 2006), al orden y desorden organizador (Morín, 1981), se identifican cuatro procesos fundamentales: explotación, conservación, destrucción y reorganización (Figura 1) (Holling \& Gunderson, 2002).

La fase de explotación se caracetiza por estrategias $r$ donde progresivamente se acumula el capital (económico, social, ecológico,etc.) para posteriormente mantenerlas gracias a las estrategias $k$ en la fase de conservación. En un sistema socio-ecológico como el presentado en Baños, la dinámica de acumulación de capital social, económico, institucional, de infraestructura y comunitario (Cutter et al., 2010), resultado de la transición de la fase $r$ a $K$ se evidencia posterior a la erupción del Tungurahua entre 1916-1918 lo que causo un alto impacto en la población (FreireGuevara, 2001). Para 1941 el pequeño caserío tendría una estructura política de nivel municipal, además que debido a su situación geográfica, que la sitúa como paso obligado desde las principales 
ciudades del centro del país hacia la amazónia, desarrollaría actividades económicas basadas en el comercio y el turismo. Los sistemas ecológicos, muchos de ellos destruidos por la erupción, se recuperan dando como resultado extensas zonas de bosques que albergarían a nueve ecosistemas distribuidos en una extensión de $1.066 \mathrm{~km} 2$ con una alta riqueza de flora y fauna (GAD del cantón Baños de Agua Santa , 2015).

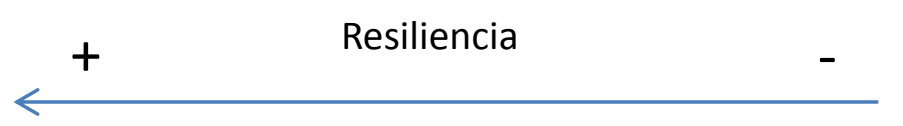

Dinámica de los Sistemas Adaptativos

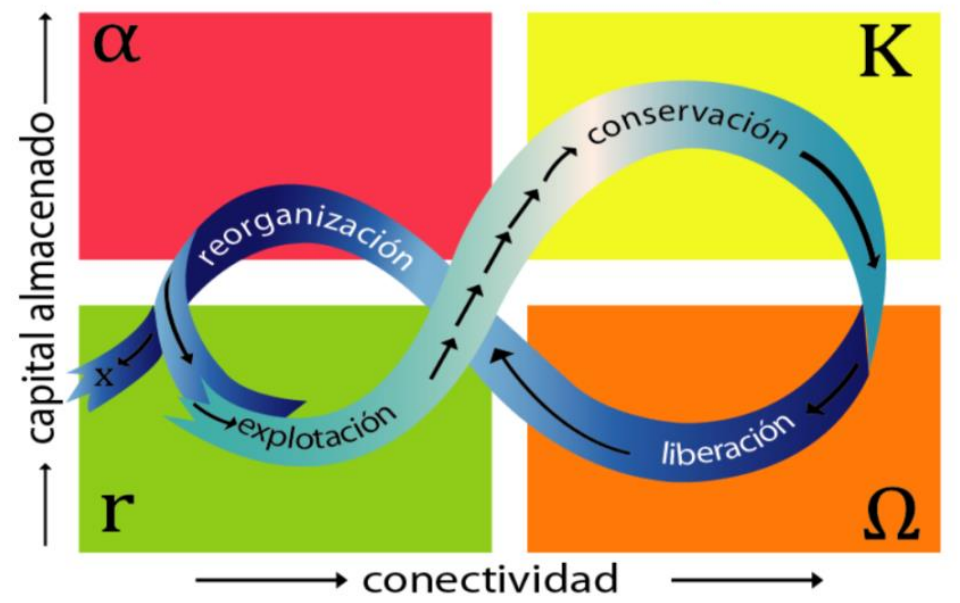

Figura 1. Representación gráfica del modelo heurístico de Panarquía

Nota: Gunderson \& Holling, Panarchy: Understanding Transformations in Human and Natural System, (2002)

Para 1990, Baños registraba una población de 15.416 habitantes (INEC, 2010), y una estabilidad en su desarrollo socio-económico acorde a las condiciones del Ecuador, la actividad que hasta ese entonces dinamizaba la economía era el turismo de tipo religioso y termal, de acuerdo a los pobladores, actividades específicas de turismo de alta montaña atraían al visitante internacional y dejaban importantes ingresos a la población; hasta 1999 la riqueza del capital ecológico, económico y social habría generado un estado de estabilidad en la región que empezaría a consolidar la fase $K$ presente en el modelo de panarquía. Para julio de 1999 aparecen las primeras señales de reactivación del Tungurahua (Aguilera \& Dueñas, 2007) la población empezó a convulsionarse y la incertidumbre fue la principal característica visible, los cerca de 80 años de relativa calma frente al fenómeno natural habían incrementado la estabilidad, pero a la vez redujeron la resiliencia, incrementando la vulnerabilidad (Schneider, 2007).

Para el 16 de octubre de 1999 se declara la "alerta naranja" y Baños sufre una evacuación forzosa que obliga a sus pobladores a adaptarse a las nuevas condiciones de deslocalización, desintegración familiar, pérdida de fuentes de ingreso, precarias condiciones en los albergues temporales, entre otras (Tobin \& Whiterford, 2002; Whiteford et al., 2013). El 5 de enero del año 2000 a pesar de la negativa de las autoridades y luego de varios enfrentamientos con la fuerza pública, la población organiza su retorno y decide retomar sus actividades productivas, sin embargo el impacto del desastre sumando al inadecuado manejo de la crisis provocarían que Baños no pueda retornar a las condiciones previas a la erupción.

La situación de Baños antes del desastre, relacionada con la etapa de $r$ a $K$ configura una fase lenta de crecimiento y acumulación, la erupción y la evacuación forzosa generaron un impacto al 
azar que perturbó el sistema socio-ecológico (Gunderson \& Holling, 2002) obligándolo a reorganizarse, dando como resultado una fase de "destrucción creativa" (Schumpeter, 1942) representada en el modelo de panarquía en la etapa de $K$ a $\Omega$. La vulnerabilidad estructural provoca la crisis que parte con una baja resiliencia en $K$ y alta conectividad entre los elementos de los diferentes ciclos panárquicos, los sistemas social y económico afectados son gestionados por algunos pobladores de la ciudad promoviendo la auto-organización de la comunidad.

La reorganización, es una fase rápida que conduce a la renovación de $\Omega$ a $\alpha$; sobre la base del legado del sistema antiguo se incrementa la incertidumbre y progresivamente se acumula el capital (ecológico, económico y social), se disminuye la conectividad y se incrementa la resiliencia; en esta fase la influencia de los factores exógenos es alta y es el escenario propicio para las innovaciones. A partir del retorno de los pobladores a la ciudad de Baños, a cuenta y riesgo de su decisión, se evidencian procesos de auto-organización; el sistema educativo y de salud que el gobierno no les proporcionaba fue tomado bajo la responsabilidad de los habitantes, se promovió la organización civil, tal es el caso de "Hermanad Baneña" y "Ojos del Volcán" que guiaron el retorno de la población y promovieron sistemas de alerta temprana, ya que el volcán se mantenía en proceso de erupción. La población organizada creó las denominadas "ollas comunales" que atendieron las necesidades de alimentación de algunos pobladores.

En la fase $\alpha$, la alta resiliencia y baja conectividad entre los elementos de cada ciclo panárquico generan la oportunidad para la configuración de nuevas estructuras que pueden generar procesos inesperados de crecimiento (Gunderson \& Holling, 2002; Holland,1995). Luego de consolidado el retorno a la ciudad, la necesidad de la reactivación económica era inminente, el turismo religioso y termal no generaba visitantes, sin embargo la experiencia de algunos empresarios identificaría una nueva forma de hacer turismo, basada en el repulsor principal de la zona, el "riesgo". Identificando las capacidades territoriales generaron un nuevo tipo de turismo basado en la aventura y los deportes extremos, que se complementaban con avistamientos del volcán en erupción desde zonas seguras, Baños había generado una oportunidad de desarrollo en el desastre. El 58.5\% de los empresarios tomaron la decisión de hacer mejoras y correcciones en sus productos y servicios y un $45.6 \%$ consideraron que la generación de nuevos productos y servicios era el camino más adecuado para superar los efectos del desastre. El nuevo sistema reorganizado se hizo posible, manteniendo la memoria de su antecesor como elemento modular que permitió la adaptación de la comunidad a las nuevas condiciones socio-económicas.

\section{SISTEMAS ADAPTATIVOS COMPLEJOS (SAC) Y LA RESILIENCIA EN LOS DESASTRE NATURALES}

Entorno al desarrollo teórico y empírico que se posee, se pueden identificar una serie de elementos que son parte esencial del concepto de resiliencia, que la caracterizan y que por lo tanto permiten diferenciar a sistemas resilientes de otros que no lo son. Sin establecer aún una jerarquía o peso ponderado, ni sus relaciones de dependencia, a manera de una primera aproximación; se los entenderá como "capacidades y factores determinantes", dentro de este contexto, se pueden definir cuatro capacidades fundamentales que presentan los sistemas adaptativos complejos y que son inherentes a la resiliencia, así: la capacidad de aprendizaje; la capacidad de auto-organización, la adaptabilidad y la capacidad de transformación (Adger et al., 2005; Gall, 2013; Gunderson, 2000; 
Walker et al., 2004). De la misma manera se pueden identificar cuatro factores críticos que son determinantes para la resiliencia en un sistema adaptativo complejo, estos son: la redundancia, la diversidad, la modularidad y la apertura (Angeler et al.,2013; Carpenter et al., 2012; Walker \& Salt, 2012).

La particularidad del estudio de la dinámica de la resiliencia en los sistemas socio económicos, entendidos como adaptativos y complejos, cuando se presenta el impacto de perturbaciones originadas por desastres naturales se lo puede encontrar en diferentes casos de estudio (Ainuddin \& Routray, 2012; Carpenter A. , 2014; Islam et al, 2013; Joerin et al., 2012; Orencio \& Fujii, 2013; Sherrieb et al., 2010; Singh-Peterson et al., 2014) su tratamiento a nivel empírico es amplio y aún carece de concesos, sin embargo se identifican tres dimensiones integradas que son recurrentes en estos análisis: ecológica, social y económica, aún más amplia es la propuesta de Cutter (2008) cuando incorpora a éstas las dimensiones: institucional, de infraestructura y competencias comunitarias. Estos esfuerzos teóricos y empíricos han hecho uso de técnicas provenientes de la teoría de la probabilidad, la indexación y las evaluaciones cualitativas (Gall, 2013).

\subsection{Capacidades de los SAC que promueven la resiliencia}

Existen diferentes capacidades de los SAC que promueven la resiliencia, se identifican así: la capacidad de aprendizaje, la auto-organización, la adaptabilidad y como una respuesta extrema al impacto de una perturbación, la capacidad del sistema para transformarse (Walker \& Salt, 2012), una síntesis de sus definiciones así como los principales autores que han contribuido a la literatura científica, se detallan en el cuadro 1.

Cuadro 1. Capacidades de los SAC

\begin{tabular}{|l|l|}
\hline \multicolumn{1}{|c|}{ CAPACIDAD } & \multicolumn{1}{c|}{ DEFINICIÓN } \\
\hline Aprendizaje & $\begin{array}{l}\text { Proceso social que promueve la diversidad de adaptaciones, la cohesión social y los } \\
\text { mecanismos de acción colectiva resultado del cual se genera conocimiento que se } \\
\text { acumulará a través de la memoria individual y colectiva. (Adger et al., 2005; Folke, 2006; } \\
\text { Pasol, 2014) }\end{array}$ \\
\hline Auto-organización & $\begin{array}{l}\text { Creación espontanea de un patrón global coherente a partir de interacciones locales } \\
\text { entre componentes inicialmente independientes, luego del efecto dinámico de } \\
\text { perturbaciones aleatorias. (Allen et al., 2014; Ashby, 1957; Heylighen, 2001) }\end{array}$ \\
\hline Adaptabilidad & $\begin{array}{l}\text { Reproducción dinámica de holgura que permite la acomodación de un sistema luego de } \\
\text { asimilar el impacto de perturbaciones. (Grabher \& Stark, 1997; Stark, 2014; Smit \& } \\
\text { Wandel, 2006) }\end{array}$ \\
\hline Transformación & $\begin{array}{l}\text { Capacidad para crear un nuevo sistema cuando las condiciones ecológicas, económicas } \\
\text { o sociales hacen que este sea insostenible. (Walker et al., 2004) }\end{array}$ \\
\hline
\end{tabular}

\subsection{Factores críticos de la resiliencia}

Los factores determinantes de la resiliencia y su incidencia en los sistemas socio-ecológicos se sistematizan en el siguiente cuadro: 
Cuadro 2. Factores críticos de la resiliencia

\begin{tabular}{|l|l|}
\hline \multicolumn{1}{|c|}{$\begin{array}{c}\text { FACTOR } \\
\text { CRÍTICO }\end{array}$} & \multicolumn{1}{c|}{ DEFINICIÓN } \\
\hline Redundancia & $\begin{array}{l}\text { Cuantificación de la existencia de elementos, sistemas u otras unidades de análisis que son } \\
\text { sustituibles entre sí (elementos de diferentes grupos funcionales), siendo capaces de } \\
\text { satisfacer los requisitos funcionales en caso de interrupción, degradación o pérdida de } \\
\text { funcionalidad. (Bellwood et al., 2004; Bruneau et al., 2003; Peterson et al., 1998) }\end{array}$ \\
\hline Diversidad & $\begin{array}{l}\text { Cuantificación de tipos o especies de elementos (mezcla) dentro de un mismo grupo } \\
\text { funcional para responder a diferentes tipos de perturbaciones, a través de una gama de } \\
\text { diferentes tipos de respuesta. (Carpenter et al., 2012; Folke, 1996; Wilson, 1992; Walker \& } \\
\text { Salt, 2012) }\end{array}$ \\
\hline Modularidad & $\begin{array}{l}\text { Subrutinas o procesos que actúan como bloques de construcción que pueden ser } \\
\text { combinados para manejar nuevos escenarios, conforman nodos críticos de enlace permiten } \\
\text { la supervivencia del sistema. (Allen et al., 2005; Carpenter et al., 2012; Holland, 2006; Martin } \\
\text { \& Sunley, 2013) }\end{array}$ \\
\hline Apertura & $\begin{array}{l}\text { Capacidad de los elementos del sistema para interconectarse con otros, estará definida por } \\
\text { la fuerza de la conexión que existe entre diferentes sistemas, lo que permitirá adopción de } \\
\text { nuevos supuestos básicos de funcionamiento. (Carpenter et al., 2012; Walker \& Salt, 2012; } \\
\text { Proag, 2014) }\end{array}$ \\
\hline
\end{tabular}

\subsection{Dimensiones básicas de análisis de la resiliencia en desastres naturales}

El impacto de los desastres naturales (terremotos, erupciones volcánicas, inundaciones, etc.) sobre los sistemas sociales y económicos se presenta como una perturbación en sus dinámicas, éste se origina en jerarquías y escalas superiores (sistemas ecológicos) y se transmite a sistemas panárquicos de escalas inferiores (sistemas sociales y económicos) de una región, se produce una conmoción de escala cruzada que obliga la generación de ciclos adaptativos que eviten el colapso del macro sistema. El sistema ecológico donde se origina el desastre, necesitará de varios años e incluso siglos para adaptarse y recuperarse mientras que los sistemas sociales y económicos requerirán de respuestas rápidas frente a la catástrofe; la gestión de desastres como una derivación de la gestión adaptativa (Folke et al., 2002) es necesaria, por lo que las dimensiones para manejar las incertidumbre generada por amenazas de orden natural deben ser identificadas y definidas, diversos estudios entorno a desastres han considerado un conjunto de variables que dotan de redundancia, diversidad, modularidad y apertura a un sistema socio-económico para que no colapse frente a una catástrofe. En la cuadro 3 se observa que las variables como sus operadores para el análisis de la resiliencia en zonas afectadas por desastres naturales son cualitativas y cuantitativas. 
Cuadro 3. Dimensiones y variables de la resiliencia

\begin{tabular}{|c|c|c|c|c|}
\hline $\begin{array}{l}\text { Dimensión/varia } \\
\text { ble }\end{array}$ & Operador & Incidencia & Justificación & $\begin{array}{l}\text { Fuente de } \\
\text { datos }\end{array}$ \\
\hline \multicolumn{5}{|l|}{ Económica } \\
\hline $\begin{array}{l}\text { Diversidad } \\
\text { económica }\end{array}$ & $\begin{array}{l}\text { Índice de diversidad } \\
\text { económica }\end{array}$ & $\begin{array}{l}\text { La diversidad de actividades económicas incrementa la } \\
\text { diversidad de oportunidades para que el sistema } \\
\text { afectado por un desastre natural pueda reestablecerse. }\end{array}$ & $\begin{array}{l}\text { (Sherrieb et al., } \\
\text { 2010) }\end{array}$ & Censo \\
\hline
\end{tabular}

Este operador tiene dos lecturas, la primera cuando la especialización en una estrecha base económica, hace que la región sea susceptible a más de las perturbaciones externas a las propias de su sector, lo que genera pocas probabilidades de reorientar su economía y limita las rutas alternativas para la recuperación.

Especialización Índice de especialización sectorial
Si la especialización se da al interior de un clúster en una industria en crecimiento, existe la posibilidad que se incremente la resiliencia cuando el sistema se ve afectado por perturbaciones externas. Cada subsistema especializado provee de modularidad y diversidad al sistema económico, desarrollando redes de cooperación e intercambio al interior del territorio lo que les permite beneficiándose de externalidades y recursos específicos (milieu territorial).

\begin{tabular}{ll}
\hline $\begin{array}{l}\text { Capacidad } \\
\text { emprendedora }\end{array}$ & $\begin{array}{l}\text { Tasa de actividad } \\
\text { emprendedora } \\
\text { \% emprendimientos por } \\
\text { oportunidad }\end{array}$ \\
\hline Social & Años de escolaridad \\
\hline $\begin{array}{l}\text { Niveles de } \\
\text { educación }\end{array}$ & $\begin{array}{l}\text { \% de cobertura del sistema } \\
\text { de seguridad social } \\
\text { \% de cobertura de seguros } \\
\text { privados }\end{array}$ \\
\hline $\begin{array}{l}\text { Cobertura de } \\
\text { seguridad social }\end{array}$ & Índice de Burgdöfer \\
\hline $\begin{array}{l}\text { Edad de la } \\
\text { población }\end{array}$ &
\end{tabular}

Actitudes emprendedoras permiten a la población ser proactiva frente a situaciones económicas adversas.

Altos niveles de escolaridad incrementan la redundancia en la población, las personas pueden asumir competencias en diferentes grupos funcionales.

Altas tasas de cobertura de seguridad social y seguros privados de salud, disminuyen la vulnerabilidad física frente a un desastre.

Una población envejecida no tiene una adecuada capacidad de respuesta. Es más vulnerable frente al impacto de un desastre.

Inequidad social Índice de GIN

La inequidad social genera conflictos en la población, la capacidad de auto organización del sistema social se ve comprometida.
(Martin \& Sunley,

2013)

Censo

(Maillat, 1995)

(Ayala \& Manzano,

2014)

GEM

(Bosma et al.,2012)

(Cutter et al., 2010)

(Ifejika et al., 2014) Censo

(Cutter et al.,2010) Censo

(Cutter et al., 2010)

(Windle et al.,2011)

Censo

(Cutter et al., 2010)

(Sherrieb et al.

2010)

(Östh et al., 2015)

(Tierney \& Bruneau, 2007)

Altos niveles de pobreza disminuyen los niveles de

Pobreza

Índice de pobreza

redundancia y diversidad funcional en los sistemas socioeconómicos.

(Rodima-Taylor \&

Censo

Olwig, 2012)

(Ifejika et al., 2014)

\begin{tabular}{ll}
$\begin{array}{l}\text { Competencias } \\
\text { personales para } \\
\text { promover la } \\
\text { resiliencia }\end{array}$ & $\begin{array}{l}\text { Indicadores psicológicos } \\
\text { basado en la escala RSA } \\
\text { (Resilience Scale for } \\
\text { Adults) }\end{array}$ \\
\hline Identity & $\begin{array}{l}\text { \% de población } \\
\text { identificada con su } \\
\text { territorio. }\end{array}$ \\
\hline Institucional & $\begin{array}{l}\text { Existencia de planes de } \\
\text { prevención de riesgos } \\
\text { naturales. }\end{array}$ \\
\hline Planes de \\
prevención
\end{tabular}

Las competencias individuales fomentan actitudes colectivas tendientes a promover adaptaciones evolutivas en los sistemas sociales.

(Friborg et al., 2005) resiliencia psicológica

La identidad de la población con el territorio promueve procesos que actúan como bloques de construcción cuando el sistema social y económico se ve alterado por un desastre natural.

(Cutter et al., 2010)

(Cavallo \& Ireland,

Encuesta

2014)

Los planes de prevención establecen módulos básicos para la operación de la comunidad en el caso de ocurrencia de un desastre natural.

Coordinación de instituciones orientadas a la mitigación de Niveles de coordinación.

La coordinación permite retroalimentaciones internas y externas, en niveles similares de escala o inter escalares del sistema socio - económico, antes durante y después del impacto de un desastre.

desastres.

Indicadores psicológicos

Cohesión basado en la escala RSA

familiar

(Resilience Scale for

Adults)
Conforma el módulo más crítico de operación de una sociedad, permite articular los procesos de las funciones esenciales de un sistema.
(Cutter et al., 2010) Observación

(Cutter et al., 2010)

(Godschalk, 2003)

(Tierney \& Bruneau,

2007)

Observación

(Bruneau et al.,

2003)

Test de

(Friborg et al., 2005) resiliencia psicológica 


\begin{tabular}{|c|c|c|c|c|}
\hline $\begin{array}{l}\text { Vías de } \\
\text { comunicación }\end{array}$ & $\begin{array}{l}\text { Existencia de vías de } \\
\text { comunicación. }\end{array}$ & $\begin{array}{l}\text { Conforma el módulo más crítico de operación de la } \\
\text { infraestructura esencial de una población. }\end{array}$ & $\begin{array}{l}\text { (Islam et al., 2013) } \\
\text { (Cutter et al., 2010) }\end{array}$ & Observación \\
\hline Servicios básicos & $\begin{array}{l}\text { \% de cobertura de servicio } \\
\text { eléctrico } \\
\% \text { de cobertura de servicio } \\
\text { de telefonía móvil. } \\
\% \text { de cobertura de agua } \\
\text { potable. } \\
\% \text { de cobertura de } \\
\text { alcantarillado. }\end{array}$ & Permiten la operación física de un sistema social & $\begin{array}{l}\text { (Cutter et al., 2010) } \\
\text { (Chopra \& Khanna, } \\
\text { 2014) } \\
\text { (Zobel \& Khansa, } \\
\text { 2014) } \\
\text { (Bruneau et } \\
\text { al.,2003) }\end{array}$ & Censo \\
\hline $\begin{array}{l}\text { Infraestructura } \\
\text { sanitaria }\end{array}$ & $\begin{array}{l}\text { Número de camas por } \\
\text { cada } 10.000 \text { habitantes. }\end{array}$ & Permiten la operación física básica del sistema de salud. & $\begin{array}{l}\text { (Cutter et al., 2010) } \\
\text { (Zobel \& Khansa, } \\
2014 \text { ) } \\
\text { (Cannon, 2008) }\end{array}$ & Censo \\
\hline $\begin{array}{l}\text { Infraestructura } \\
\text { educativa }\end{array}$ & $\begin{array}{l}\text { Cobertura de educación } \\
\text { escolar y de bachillerato. }\end{array}$ & $\begin{array}{l}\text { Establecen los módulos básicos de operación social de } \\
\text { una comunidad, se promueve la redundancia y la } \\
\text { diversidad de supervivencia. }\end{array}$ & $\begin{array}{l}\text { (Tierney \& Bruneau, } \\
\text { 2007) } \\
\text { (Cannon, 2008) }\end{array}$ & Censo \\
\hline
\end{tabular}

\section{BAÑOS DE AGUA SANTA CIUDAD RESILIENTE}

La ciudad de Baños fue declarada por las Naciones Unidad en julio de 2014 como "ciudad resiliente" frente a eventos volcánicos (UNISDR, 2015), pero ¿qué factores la hacen resiliente?, en base a la propuesta de capacidades, factores críticos y dimensiones, resumidas en los cuadros 2 y 3 se realiza una primera aproximación sobre las variables que pueden ser determinantes en el estudio de la resiliencia, si bien la complejidad del tema obliga a considerar varios elementos de estudio, se presenta esta propuesta de inputs que podrán ser tratados con una serie de herramientas que son utilizadas por la ciencia de la complejidad (Gall, 2013).

Este estudio hace uso de las fuentes de datos de los Censos de Población y Vivienda (2010) y el Censo Nacional Económico (2010), además utiliza los resultados preliminares de la aplicación del cuestionario RSA (Resilience Scale for Adults) a 290 jefes de hogares y la aplicación de una encuesta que analiza la percepción de 316 empresarios de la ciudad de Baños, en las variables de corte cualitativo se incorporaron las respuestas de 26 entrevistas a profundidad a líderes locales, académicos y empresarios.

\subsection{Dimensión económica}

\subsection{1 Índice de diversidad económica}

Considerando el concepto del Índice de Herfindahl - Hirschman (IHH), en base a los sectores económicos se determina el nivel de concentración sectorial en la ciudad de Baños.

Tabla 1. Sectores económicos del cantón Baños

\begin{tabular}{|c|c|c|}
\hline $\begin{array}{l}\text { Sectores Económicos } \\
\text { Código CIIU4 - Sección (1 dígito) } 2013\end{array}$ & $\begin{array}{l}\text { Total empresas } \\
\text { registradas }\end{array}$ & Fracción \\
\hline Agricultura, ganadería, silvicultura y pesca & 212 & $6.73 \%$ \\
\hline Explotación de Minas y Canteras & 5 & $0.16 \%$ \\
\hline Industrias Manufactureras & 190 & $6.03 \%$ \\
\hline Comercio & 1,077 & $34.19 \%$ \\
\hline Construcción & 40 & $1.27 \%$ \\
\hline Servicios & 1,626 & $51.62 \%$ \\
\hline Total & 3,150 & $100 \%$ \\
\hline
\end{tabular}




$$
H=\sum_{i=1}^{n} S_{i}^{2} ; \text { donde } i=1 \ldots n .=3911.27
$$

La economía en la ciudad de Baños está altamente concentrada en dos sectores económicos: comercio y servicios.

\subsubsection{1 Índice de especialización sectorial (IES)}

Para el cálculo del índice de especialización sectorial se utilizará como fuente las cuentas regionales publicadas por el Banco Centra del Ecuador (BCE, 2015); a partir de esta fuente se calcula el índice de especialización sectorial por sectores y se obtiene el respectivo promedio para lo cual se emplea la siguiente fórmula:

$$
I E S=\frac{\frac{V i j}{\sum_{j=1}^{n} V i j}}{\frac{\sum_{i=1}^{n} V i j}{\sum_{i=1}^{n} \sum_{j=1}^{n} V i j}}
$$

De donde:

V= variable de análisis

Vij= Valor de la variable V correspondiente al empleo sector "i” y región “j”

$\mathrm{V} s \mathrm{j}=\sum_{j=1}^{n} V s$ Valor de $\mathrm{V}$ correspondiente al total sectorial i.

Vir $=\sum_{j=1}^{n} V s$ Valor de $\mathrm{V}$ correspondiente al total regional de la región j.

$\operatorname{Vsr} \sum_{i=1}^{n} \sum_{i=1}^{n} V i j$ Valor de $\mathrm{V}$ correspondiente al total global (suma regional).

Para el caso de Baños los índices de especialización sectorial son:

Tabla 2. Índices de especialización sectorial - Baños

\begin{tabular}{ll}
\hline $\begin{array}{l}\text { Agricultura, ganadería, } \\
\text { silvicultura y pesca }\end{array}$ & $\mathbf{0 . 3 6}$ \\
\hline $\begin{array}{l}\text { Explotación de Minas y } \\
\text { Canteras }\end{array}$ & 0.19 \\
\hline Industrias Manufactureras & 0.86 \\
\hline Comercio & 0.84 \\
\hline Construcción & 0.22 \\
\hline Servicios & $\mathbf{1 . 2 8}$ \\
\hline
\end{tabular}

Los datos evidencian una especialización de Baños en el sector servicios, su orientación histórica corresponde a la prestación de servicios turísticos.

\subsubsection{Capacidad emprendedora}

Baños hasta 2013 registra 3.150 empresas de las cuales, el 95\% corresponden a microempresas y generan el $3.20 \%$ de ingresos del cantón, la mediana y gran empresa que representan el $0.54 \%$ y el $0.12 \%$ respectivamente generan el $67.3 \%$ de los ingresos. De acuerdo al Global Entreneurship Monitor - GEM (Lasio et al.,2014), Ecuador presenta un $34.9 \%$ de emprendimientos motivados por la oportunidad de mejora (el más bajo de la región) frente a un 29.4\% motivados por la necesidad (uno de los más altos de región), en la encuesta aplicada en el 
cantón baños bajo los criterios del GEM, los resultados presentan que el $80.70 \%$ de las empresas establecidas en los últimos 10 años se crearon motivadas por la oportunidad de negocio, mientras que el $19.30 \%$ están relacionadas con otros motivos entre ellos la necesidad. Los datos se contraponen con los resultados del GEM a nivel nacional, identificando a los empresarios de Baños fuertemente motivados por la oportunidad antes que por la necesidad.

\subsection{Dimensión Social}

\subsubsection{Nivel de escolaridad}

Para esta variable se considerara el año de escolaridad acorde al Censo de Población y Vivienda (2010); los años de escolaridad que presenta el cantón Baños son de 9,6; este valor es cercano al presentado por la provincia de Pichincha (10,6 años) que es el más alto del país.

\subsubsection{Cobertura de seguridad social}

La seguridad social estatal cubre al $23.77 \%$ de los pobladores del cantón mientras que el dato a nivel de la provincia de Tungurahua es de $18.42 \%$ y a nivel nacional la cobertura es del 21.67\%. En Baños el $8.07 \%$ de pobladores tiene seguro de salud privado, para la provincia de Tungurahua éste valor es de 6.03\%, mientras que a nivel país es del 9.35\% (Censo de Población y Vivienda, 2010).

\subsubsection{Edad de la población}

La cualificación de la edad de la población se la determinara en base al índice de Burgdofer, la fuente de información es el Censo de Población y Vivienda (2010), el cual proporciona los siguientes datos para su cálculo:

Tabla 3. Distribución poblacional por rango de edades - Baños

\begin{tabular}{crcc}
\hline \multicolumn{1}{c}{ Edad } & Valor & Total & Porcentaje \\
\hline De 5 a 9 años & 1809 & \multirow{2}{*}{3661} & \\
De 10 a 14 años & 1852 & & $18,29 \%$ \\
De 45 a 49 años & 1069 & & \\
De 50 a 54 años & 872 & \multirow{2}{*}{3271} & \\
De 55 a 59 años & 730 & & $16,34 \%$ \\
De 60 a 64 años & 600 & & 120101 \\
\hline
\end{tabular}

Fuente: Censo de Población y vivienda (2010).

Índice de Burgdofer $=\frac{\text { Porcentaje de la poblacion de } 5 \text { a } 14 \text { años }}{\text { Porcentaje de la población de } 45 \text { a } 64 \text { años }}=1,12$

Un valor de superior a 1 en este índice, establece que la población es joven.

\subsubsection{Desigualdad}

Para el análisis de la desigualdad se considera el coeficiente de Gini. 
Tabla 4. Componentes del Índice de Gini

\begin{tabular}{clrrrrrrr}
\hline Estrato & Ingresos medios anuales & \multicolumn{1}{c}{ xi } & \multicolumn{1}{c}{$\mathrm{ni}$} & $\mathrm{Ni}$ & $\mathrm{pi}$ & \multicolumn{1}{c}{ ui } & Ui & qi \\
\hline 1 & De 1 a 9999 dólares & 5000 & 799 & 799 & 0,551 & 3995000 & 3995000 & $13,91 \%$ \\
2 & De 10000 a 29999 & 19999,5 & 453 & 1252 & 0,863 & 9059773,5 & 13054773,5 & $45,46 \%$ \\
3 & De 30000 a 49999 & 39999,5 & 96 & 1348 & 0,929 & 3839952 & 16894725,5 & $58,84 \%$ \\
4 & De 50000 a 69999 & 59999,5 & 25 & 1373 & 0,946 & 1499987,5 & 18394713 & $64,06 \%$ \\
5 & De 70000 a 89999 & 79999,5 & 13 & 1386 & 0,955 & 1039993,5 & 19434706,5 & $67,68 \%$ \\
6 & Más de 89999 & 144999,5 & 64 & 1450 & 1 & 9279968 & 28714674,5 & $100,00 \%$ \\
\hline
\end{tabular}

Fuente: Censo Económico INEC (2010)

Reemplazando los valores en la fórmula del coeficiente el resultado es de:

$$
G=\frac{\sum_{i=1}^{K-1}(p i-q i)}{\sum_{i=1}^{k-1} p i}=0,41
$$

La ciudad de Baños mantiene niveles de desigualdad moderada, mientras que el resultado a nivel país se encuentra para el periodo 2010 -2014 en 0,46; también dentro del mismo nivel.

\subsection{5 Índice de pobreza (para países en vías de desarrollo)}

Para el índice de pobreza se utiliza la fórmula (5) y se aplica a la ciudad de Baños y a nivel país.

$$
\text { HPI } 1=\left[\frac{1}{3}\left(P_{1}^{\alpha}+P_{2}^{\alpha}+P_{3}^{\alpha}\right)\right]^{\frac{1}{\alpha}}
$$

$\mathrm{P}_{1}$ : Probabilidad al nacer de no sobrevivir la edad de 40 años

$\mathrm{P}_{2}$ : Tasa de analfabetismo adulto.

$\mathrm{P}_{1}$ : Población sin acceso sostenible a una fuente de agua tratada.

$\alpha: 3$

\begin{tabular}{|c|c|c|}
\hline Índice de pobreza & Baños & Nacional \\
\hline Tasa analfabetismo & $3,74 \%$ & $5.16 \%$ \\
\hline Población sin acceso agua tratada & $17,78 \%$ & $28,02 \%$ \\
\hline Probabilidad de no sobrevivir 40 años & $4,10 \%$ & $10.2 \%$ \\
\hline HPI1 & $12,41 \%$ & $19,77 \%$ \\
\hline
\end{tabular}

Tabla 4. Componentes del índice de pobreza

El índice de pobreza de la ciudad de Baños en menor que el registrado a nivel nacional, sobre la base de los datos utilizados en la fórmula (5).

\subsubsection{Competencias personales para la resiliencia}

De acuerdo a la encuesta RSA (Resilience Scale for Adults) aplicada a 290 jefes de familias del cantón Baños con un valor de confiabilidad dado por un Alfa de Cronbach de 0.822 , se obtuvo los siguientes resultados, en las variables que evalúan las competencias personales para la resiliencia: 
Tabla 5. Medias de las variables para competencias profesionales RSA

\begin{tabular}{llr}
\hline \multicolumn{3}{c}{ Estadísticos descriptivos } \\
\hline & Media & Varianza \\
\hline VAR01 & 4.2276 & 1.353 \\
\hline VAR02 & 4.2276 & 1.353 \\
\hline VAR03 & 4.4241 & 1.006 \\
\hline VAR04 & 3.8448 & 1.619 \\
\hline VAR05 & 3.9862 & 1.882 \\
\hline VAR06 & 3.6724 & 1.757 \\
\hline VAR07 & 4.0655 & 1.459 \\
\hline VAR08 & 4.1138 & 1.520 \\
\hline VAR09 & 3.8793 & 1.643 \\
\hline VAR10 & 4.0069 & 1.557 \\
\hline & $\bar{X}=4.0448$ & \\
\hline
\end{tabular}

El valor obtenido del promedio de las medias de las variables analizadas indica que existen competencias personales para la resiliencia en los jefes de familia de la ciudad de Baños.

\subsubsection{Identidad territorial}

La identidad territorial es determinada a través de una pregunta aplicada a 290 jefes de familia de la ciudad de Baños que indaga sobre ¿qué tan orgulloso se siente de vivir en Baños? y un bloque de siete categorías relacionadas con la identidad territorial, todo este análisis hace uso de escalas de Likert.

Las siete categorías evaluadas son: logros económicos, capacidad de organización ciudadana, sistema de prevención de riesgos, autoridades cantonales, autoridades del gobierno central, infraestructura e historia; los encuestados valoran a la ciudad de Baños en una escala de 1 a 4 con 3.04; valor que integra los resultados de las calificaciones dadas a cada categoría evaluada. Las categorías evaluadas presentan un nivel de confiabilidad (Alfa de Cronbach) de 0,724. Un valor superior a 2 indica la existencia de una percepción positiva sobre su identidad territorial. Las categorías que inciden positivamente en la evaluación de la identidad territorial son: la historia de la ciudad de Baños, sus logros económicos, su infraestructura y capacidad de organización.

Tabla 5. ¿Qué tan orgulloso se siente de vivir en Baños?

Frecuencia Porcentaje

\begin{tabular}{lcc}
\hline No está orgulloso & 4 & $1.38 \%$ \\
\hline No tan orgulloso & 11 & $3.79 \%$ \\
\hline Orgulloso & 55 & $18.97 \%$ \\
\hline Muy orgulloso & 220 & $75.86 \%$ \\
\hline Total & 290 & $100.00 \%$ \\
\hline
\end{tabular}




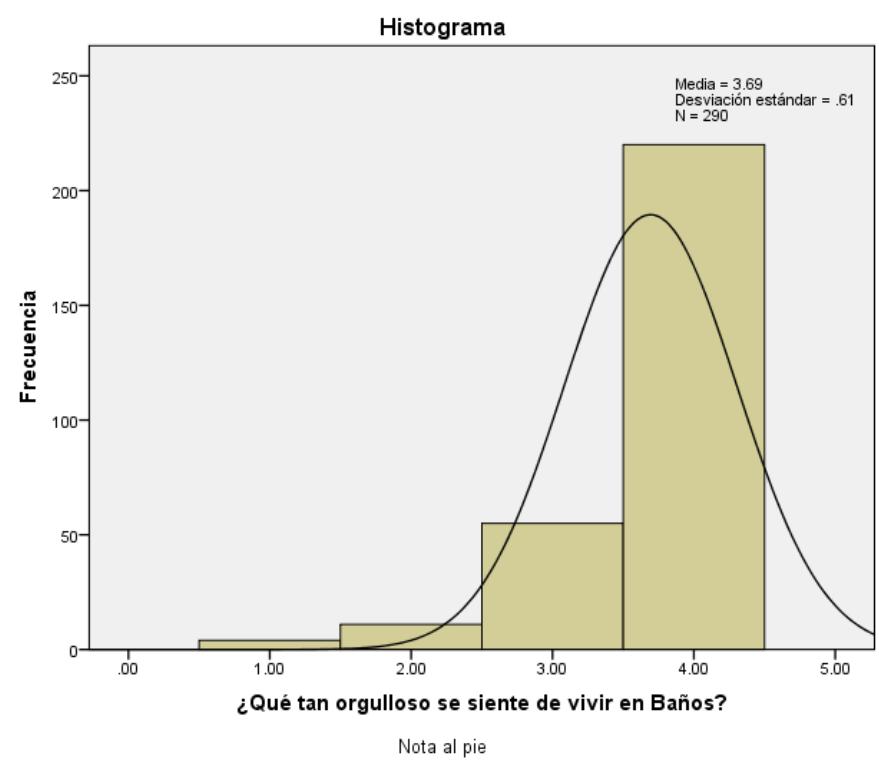

Figura 2. ¿Qué tan orgulloso se siente de vivir en Baños?

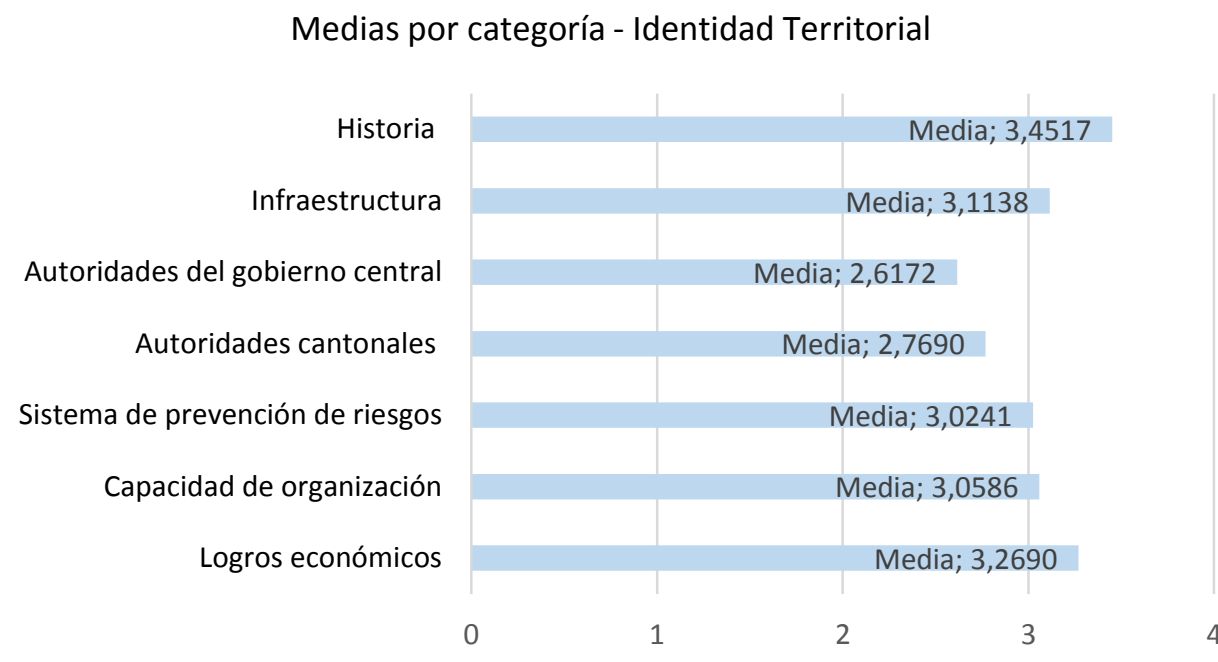

Figura 3. Valoración de las categorías - Identidad territorial en Baños

\subsection{Dimensión Institucional}

\subsubsection{Planes de prevención}

La evaluación de esta variable se la realiza a través de la verificación in situ de los planes de prevención por parte de los organismos encargados de la gestión de riesgos. Para el caso de Baños de Agua Santa, existen planes de prevención que han sido diseñados y socializados considerando la realidad del territorio. El $83.9 \%$ de las empresas y el $75.9 \%$ de la población han participados en simulacros relacionados con el volcán Tungurahua. 


\subsubsection{Coordinación de instituciones orientadas a la mitigación de desastres.}

La evaluación de esta variable se la realiza a través de un proceso de verificación in situ, considerando los siguientes criterios:

- Existe un organismo coordinador de gestión de riesgos

- El organismo coordinador incorpora sistemas operativos para la prevención y mitigación de desastres.

- El organismo coordinador incorpora a representantes de la sociedad civil en la toma de decisiones.

- El organismo coordinador cuenta con recursos económicos para la prevención y mitigación de desastres.

Para el caso de Baños de Agua Santa y sobre la base de la legislación del Ecuador, se ha conformado el Comité de Operaciones de Emergencia (COE) a nivel cantonal, el mismo que opera bajo el principio de descentralización subsidiaria, que implica responsabilidad directa de las instituciones dentro de su ámbito geográfico, incorpora autoridades seccionales, autoridades de organismos de mitigación de riesgos, técnicos especialistas y representantes de la sociedad civil, en caso de desastres se integrará con el COE provincial y nacional.

\subsubsection{Cohesión familiar}

De acuerdo a la encuesta RSA (Resilience Scale for Adults) aplicada a 290 familias del cantón Baños con un valor de confiabilidad de Alfa de Cronbach de 0.775 , se obtuvo los siguientes resultados, en las variables que evalúan la cohesión familiar:

Tabla 6. ¿Qué tan orgulloso se siente de vivir en Baños?

\begin{tabular}{rrr}
\hline \multicolumn{3}{c}{ Estadísticos descriptivos } \\
\hline & Media & Varianza \\
\hline VAR21 & 3.9345 & 1.231 \\
\hline VAR22 & 4.5828 & .915 \\
\hline VAR23 & 4.1345 & 1.501 \\
\hline VAR24 & 4.3931 & .925 \\
\hline VAR25 & 4.2276 & 1.422 \\
\hline VAR26 & 3.6310 & 2.386 \\
\hline $\bar{X}$ & $=4.1506$ \\
\hline
\end{tabular}

El valor obtenido del promedio de las medias de las variables analizadas indica que existe una alta cohesión familiar en la ciudad de Baños.

\subsection{Dimensión Infraestructura}

\subsubsection{Vías de comunicación}

Las vías de comunicación son "líneas de vida" que permiten, a más de la conexión con otras zonas geográficas, el establecimiento de rutas de evacuación y el abastecimiento en caso de 
desastre. La ciudad de Baños cuenta en la actualidad con dos vías de comunicación con el resto del territorio nacional, lo que le vuelve vulnerable frente a un desastre de grandes magnitudes. La irregularidad geográfica es la principal causa de la ausencia de infraestructura vial, la vía más importante la constituye la ruta Ambato - Baños - Puyo y la vía que actuaría como emergente es la ruta Ambato - Patate - Baños; la primera dentro de la zona de riesgo por presencia de lahares del volcán Tungurahua y la segunda dentro de una zona de riesgo por movimientos de masa. Si los dos eventos naturales se darían de forma simultánea, Baños quedaría incomunicada con el resto del país.

\subsection{Servicios básicos}

La cobertura de energía eléctrica en las viviendas de la ciudad de Baños es del $98.1 \%$; frente al total de la cobertura provincial que registra el $96,75 \%$ y a nivel nacional que registra un valor de 93.19\%.

El porcentaje de viviendas con eliminación de aguas servidas a través de la red pública de alcantarillado en Baños es del 78.72 \% frente a los datos de cobertura provincial del $61.9 \%$ y nacional que se sitúan en el 53.59\%.

Las viviendas con abastecimiento de agua por red pública en su interior en Baños se sitúan en el $73.85 \%$, mientras que el dato a nivel provincial es del $60.32 \%$ y el nacional se ubica en torno al $60.15 \%$.

Respecto a la comunicación vía móvil, el 78.09\% de la población de Baños dispone de este servicio, a nivel provincial el resultado es del $71.52 \%$ y el $76.28 \%$ a nivel nacional.

COBERTURA DE SERVICIOS

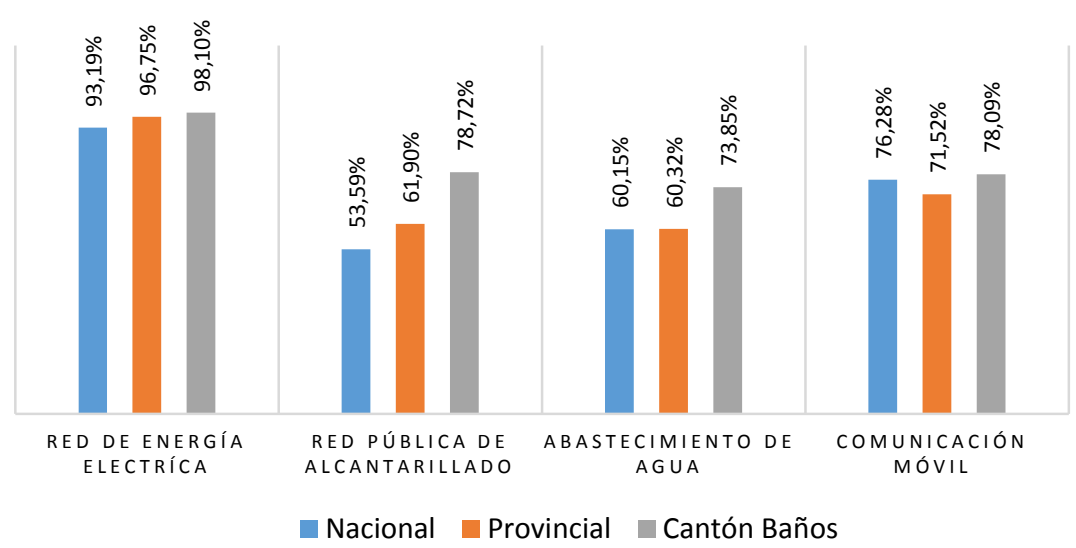

Figura 4. Comparativo de cobertura de servicios básicos para Baños.

La cobertura entono a los servicios analizados en la ciudad de Baños, es superior en todos los casos al promedio provincial y nacional.

\subsubsection{Infraestructura sanitaria}

Se analiza el número de camas disponibles por cada 10.000 habitantes, Baños registra un valor 8.03, a nivel provincial la cifra es de 15,79 y 15,36 a nivel nacional (INEC, 2013). La infraestructura es deficiente y no cubre la demanda existente en la población. 


\subsubsection{Infraestructura educativa}

De acuerdo a los datos del Ministerio de Educación de Ecuador a 2014, Baños cuenta con 27 instituciones educativas entre centros de educación inicial, educación básica y bachillerato, con un total de 5.411 estudiantes matriculados, considerando que la población en edades escolares entre 5 y 19 años es de 5.963, se puede establecer que la capacidad de infraestructura instalada en el cantón no cubre su demanda educativa.

Cada una de las dimensiones y variables analizadas tienen un efecto positivo, negativo o neutral sobre la resiliencia de Baños de Agua Santa, frente al impacto generado por la erupción del volcán Tungurahua, esto se resumen en el cuadro 5.

Cuadro 4. Impacto de los factores críticos de la resiliencia en Baños de Agua Santa

\begin{tabular}{|c|c|c|}
\hline Dimensión/variable & Análisis & Incidencia sobre la resiliencia \\
\hline \multicolumn{3}{|l|}{ ECONÓMICA } \\
\hline Diversidad Económica & $\begin{array}{l}\text { La economía de la ciudad de Baños es } \\
\text { altamente concentrada, lo que la hace } \\
\text { vulnerable al impacto de desastres } \\
\text { naturales. }\end{array}$ & $\begin{array}{l}\text { Negativa } \\
\text { Disminuye la diversidad. }\end{array}$ \\
\hline Especialización sectorial & $\begin{array}{l}\text { Existe una especialización sectorial en un } \\
\text { clúster que corresponde a una industria en } \\
\text { crecimiento, dotando a la población de } \\
\text { capacidades resilientes para la } \\
\text { recuperación económica. }\end{array}$ & $\begin{array}{l}\text { Positiva } \\
\text { Incrementa la diversidad } \\
\text { dentro del clúster. }\end{array}$ \\
\hline Capacidad emprendedora & $\begin{array}{l}\text { Existe una clara orientación hacia el } \\
\text { emprendimiento por oportunidad, la } \\
\text { población se destaca por ser } \\
\text { emprendedora lo que fortalece la } \\
\text { resiliencia del sistema económico. }\end{array}$ & $\begin{array}{l}\text { Positiva } \\
\text { Fortalece la modularidad del } \\
\text { sistema económico. }\end{array}$ \\
\hline \multicolumn{3}{|l|}{ SOCIAL } \\
\hline Niveles de Educación & $\begin{array}{l}\text { Los niveles de escolaridad son altos en } \\
\text { comparación a la región, la educación } \\
\text { permite asumir competencias en } \\
\text { diferentes grupos funcionales cuando la } \\
\text { comunidad se ve afectada por un evento } \\
\text { inesperado. }\end{array}$ & $\begin{array}{l}\text { Positiva } \\
\text { Fortalece la redundancia y } \\
\text { promueve la apertura. }\end{array}$ \\
\hline Cobertura de seguridad social & $\begin{array}{l}\text { A pesar de que la cobertura no es la } \\
\text { adecuada, se observa condiciones } \\
\text { superiores al promedio de la región. }\end{array}$ & $\begin{array}{l}\text { Positiva } \\
\text { Promueve la modularidad. }\end{array}$ \\
\hline Edad de la población & $\begin{array}{l}\text { La población es joven por lo que su } \\
\text { capacidad de respuesta frente a un } \\
\text { desastre se incrementa. }\end{array}$ & $\begin{array}{l}\text { Positiva } \\
\text { Promueve la apertura. }\end{array}$ \\
\hline Inequidad social & $\begin{array}{l}\text { La población mantiene niveles de } \\
\text { desigualdad moderada comparables al } \\
\text { promedio de la región. }\end{array}$ & Neutral \\
\hline Pobreza & $\begin{array}{l}\text { El índice de pobreza para países en vías de } \\
\text { desarrollo, aplicado a Baños es menor al } \\
\text { promedio nacional. }\end{array}$ & Positivo \\
\hline $\begin{array}{l}\text { Competencias personales para } \\
\text { promover la resiliencia }\end{array}$ & $\begin{array}{l}\text { Se identifica en los jefes de familia } \\
\text { competencias personales para la } \\
\text { resiliencia. }\end{array}$ & $\begin{array}{l}\text { Positiva } \\
\text { Se promueve la redundancia. }\end{array}$ \\
\hline Identidad & $\begin{array}{l}\text { La población se siente altamente } \\
\text { identificada con su territorio. }\end{array}$ & $\begin{array}{l}\text { Positiva } \\
\text { Facilita la modularidad. }\end{array}$ \\
\hline \multicolumn{3}{|l|}{ INSTITUCIONAL } \\
\hline Planes de prevención & $\begin{array}{l}\text { Existen planes de prevención coherentes } \\
\text { con la realidad del territorio. }\end{array}$ & $\begin{array}{l}\text { Positiva } \\
\text { Promueven la modularidad. }\end{array}$ \\
\hline $\begin{array}{l}\text { Coordinación de instituciones } \\
\text { orientadas a la mitigación de } \\
\text { desastres. }\end{array}$ & $\begin{array}{l}\text { Existen planes de mitigación de riesgos con } \\
\text { una estructura coordinada. }\end{array}$ & $\begin{array}{l}\text { Positiva } \\
\text { Establecen la acción } \\
\text { coordinada de los módulos } \\
\text { básicos de reconstrucción. }\end{array}$ \\
\hline
\end{tabular}




\begin{tabular}{lll}
\hline Cohesión familiar & Existe una alta cohesión familiar. & $\begin{array}{l}\text { Positiva } \\
\text { Promueve la modularidad. }\end{array}$ \\
\hline INFRAESTRUCTURA & & \\
\hline Vías de comunicación & $\begin{array}{l}\text { Vías de comunicación limitadas, localizadas } \\
\text { en zonas de riesgo. }\end{array}$ & $\begin{array}{l}\text { Negativa } \\
\text { Disminuye la modularidad y } \\
\text { diversidad. }\end{array}$ \\
\hline Servicios básicos & La cobertura de servicios básicos supera los & Positiva \\
& promedios de la región. & Promueve la modularidad. \\
\hline Infraestructura sanitaria & No cubre las necesidades de la población & Negativa \\
& & Disminuye la modularidad. \\
\hline Infraestructura educativa & No cubre la demanda educativa & Negativa
\end{tabular}

\section{CONCLUSIONES}

El modelo heurístico de panarquía facilita la comprensión de la compleja dinámica de los SAC, la interrelación de sus ciclos en diferentes escalas deja espacio para la innovación, el desarrollo y la evolución. El comportamiento de los sistemas socio-ecológicos equiparados como SAC en situaciones no esperadas, trae consigo un conjunto de interrogantes sobre cómo deben ser gestionados para evitar su deterioro y destrucción; esto ha motivado la búsqueda de herramientas que generen información relevante, oportuna, confiable y económica para el diseño de políticas orientadas a promover la resiliencia en zonas que potencialmente pueden sufrir el impacto de desastres naturales.

Los SAC brindan un cuerpo teórico adecuado para el análisis de la resiliencia en zonas de desastre; el aprendizaje, la auto-organización, la adaptabilidad, la trasformación son capacidades que establecen una directriz para la selección de métricas orientadas a cuantificarla. Las variables seleccionadas en este trabajo son coherentes con las capacidades de los SAC y actúan como catalizadoras de los factores críticos de la resiliencia.

El estudio sobre la ciudad de Baños de Agua Santa evidencia a una población con una capacidad dinámica de aprendizaje, adaptación y auto-organización que le ha permitido mantener sus funciones esenciales a pesar del impacto ecológico, social y económico que generaría la erupción del volcán Tungurahua, como respuesta, se vieron obligados a desarrollar nuevos productos y servicios así como también a mejorar los existentes, dentro de su principal actividad económica, el turismo. El cambio de turismo religioso y termal hacia el turismo de aventura y el ecoturismo es una clara evidencia.

Los factores que más se destacan en el perfil resiliente de la ciudad de Baños de Agua Santa son su alta especialización en el sector turístico, lo que le permitie reconstruir su tejido económico haciendo uso de la experiencia histórica de sus pobladores en una suerte de milieu territorial. La capacidad emprendedora, cuya motivación fundamental es la búsqueda de oportunidades de mejora es una característica particular de la ciudad y que se contrapone con el comportamiento de la región en la que la necesidad es la principal motivación para emprender. La estructura del sector turístico en Baños es diversa, no existe empresas dominantes y la competencia regula el mercado, esta diversidad de actores y su nivel de especialización en actividades propias de de él, dotaron de adapatabilidad al sistema socio-económico lo que facilitó la auto-organización de la población luego del desastre.

Las variables sociales analizadas en Baños, superan los promedios de su región demostrando que la dimensión social es una fortaleza intrínseca de la población, lo que le permite 
contar con un tejido social que es capaz de interrelacionarse y configurar módulos básicos que sustenten su funcionamiento a pesar de los impactos generados por un evento externo a ellos. En la actualidad la ciudad de Baños ha generado una curva de aprendizaje frente al riesgo que implica su ubicación geográfica, existen planes y programas coordinados entre las instituciones públicas, privadas y la población que responden a sus necesidades; se evidencia una alta participación y conocimiento del riesgo y sus potenciales consecuencias, lo que incrementa su resiliencia frente a un desastre.

La dimensión de estudio relacionada con la infraestructura evidencia serias deficiencias que han sido evidentes a lo largo de la historia de la ciudad, su ubicación geográfica que es una fortaleza para el desarrollo de la actividad económica de Baños, es a la vez una debilidad, ya que debido a su orografía dificulta la construcción de vías de comunicación alternativas en el caso de un desastre. Si se presentase un evento de grandes magnitudes relacionado con el volcán o movimientos de masa, la población puede quedar aislada y sin abastecimientos básicos haciéndola más vulnerable y menos resiliente.

Las variables analizadas en el caso propuesto permiten establecer un diagnóstico general de una población, que por sus características ha sido considerada por la Naciones Unidas (2014) como una referencia mundial de comportamiento resiliente frente a desastres generados por una erupción volcánica, sin embargo queda como tarea identificar otras variables de estado que permitan afinar la investigación en torno a la resiliencia frente a shocks externos, así como el análisis de las interrelaciones que estas puedan tener dentro de la dinámica de los ciclos adaptativos de los sistemas complejos. El impacto de los desastres naturales suele tomar grandes dimensiones en países pobres o en vías de desarrollo, donde los datos son limitados o inexistentes; por lo que es necesario diseñar herramientas metodológicas que permitan abordar la resiliencia haciendo uso de datos estadísticos generales y estudios cualitativos de la población, lo que demanda métodos mixtos que consideren la complejidad de las relaciones de los sistemas económico, social y ecológico, su interrelación jerárquica y escalar que tiene lugar en un entorno de incertidumbre. La aplicación de herramientas metodológicas para abordar la resiliencia en entornos dinámicos, emergentes y con información limitada es un campo prometedor para la investigación, donde por ejemplo, el análisis multicriterio difuso y el modelamiento basado en agentes ( $A B M$ ) poseen buenas perspectivas.

\section{BILIOGRAFÍA}

1. ADGER, N., HUGHES, T., FOLKE, C., CARPENTER, S., \& ROCKSTRÖM, J. (2005). Social-ecological resilience to coastal disasters. Science, 1036-1039.

2. AGUILERA, E., \& DUEÑAS, W. (2007). Las erupciones explosivas del volcán Tungurahua de julio $y$ agosto de 2006. Quito: COSUDE.

3. AINUDDIN, S., \& ROUTRAY, J. (2012). Community resilience framework for an earthqake prone area in Baluchistan. International Journal of Disaster Risk Reduction, 25-36.

4. AlLeN, C. R., ANGeler, D. G., GARMESTANI, A. S., GUNDERSON, L. H., \& HOLLING, C. (2014). Panarchy: Theory and Application. Ecosystems, 578-589.

5. ANGELER, D. G., ALLEN, C. R., \& JOHNSON, R. K. (2013). Measuring the relative resilience of subarctic lakes to global change: redundancies of functions within and across temporal scales. 
Journal of Applied Ecology, 572-584.

6. ASHBY, W. R. (1957). An Introduction to Cybernetics. London: Chapman \& Hall Ltd.

7. AYALA, J.-C., \& MANZANO, G. (2014). The resilience of the entrepreneur. Influence on the success of the business. A longitudinal analysis. Journal of Economic Psychology, 126-135.

8. BCE. (10 de Agosto de 2015). Banco Central de Ecuador. Obtenido de http://www.bce.fin.ec/index.php/component/k2/item/293-cuentas-provinciales

9. BELLWOOD, D., HUGHES, T., FOLKE, C., \& NYSTRO, M. (2004). Confronting the coral reef crisis. Nature, 827-833.

10. BOSMA, N., CODURA, A., LITOVSKY, Y., \& SEAMAN, J. (2012). A report on the design, data and quality control of the Global Entrepreneurship Monitor. London: Global Entrepreneurship Research Association.

11. BRUNEAU, M., CHANG, S. E., EGUCHI, R. T., LEE, G. C., O’ROURKE, T. D., O'ROURKE, T. D., Y OTROS. (2003). A Framework to Quantitatively Assess and Enhance the Seismic Resilience of Communities. Earthquake Spectra, 733-752.

12. CANNON, T. (2008). Reducing People's Vulnerability to Natural Hazards. Research Paper No. 2008/34 UNU-WINDER, http://www.wider.unu.edu/publications/working-papers/researchpapers/2008/en_GB/rp2008-34/.

13. CARPENTER, A. (2014). Resilience in the social and physical realms: Lessons from the Gulf Coast. International Journal of Disaster Risk Reduction, 1-12.

14. CARPENTER, S., ARROW, K., BARRETT, S., BIGGS, R., BROCK, W., \& CRÉPIN, A.-S. (2012). General Resilience to Cope with Extreme Events. Sustainability, 3248-3259.

15. CAVAlLO, A., \& IRELAND, V. (2014). Preparing for complex interdependent risk: A system of systems approach to building disaster resilience. International Journal of Disaster Risk Reduction, 181-193.

16. CHOPRA, S., \& KHANNA, V. (2014). Understanding resilience in industrial symbiosis networks: Insights from network analysis. Journal of environmental management, 86-94.

17. CUTTER, S. L., BARNES, L., BERRY, M., BURTON, C., EVANS, E., TATE, E., Y OTROS. (2008). A place-based model for understanding community resilience to natural disasters. Global Environmental Change, 598-606.

18. CUTTER, S., BURTON, C., \& EMRICH, C. (2010). Disater Resilience Indicators for Benchmarking Baseline Conditions. Journal of Homeland Security and Emergency, 1-22.

19. FOLKE, C. (2002). Social-Ecological Resilience and Behavioural Responses. Recuperado el 22 de 04 de 2014, de The Beijer Intitute of Ecological Economics: http://www.beijer.kva.se/PDF/87823499_Disc155.pdf

20. FOLKE, C. (2006). Resilience: The emergence of a perspective for social-ecological systems analyses. Global Environmental Change, 253-267.

21. FOLKE, C., HOLLING, C. S., \& PERRINGS, C. (1996). Biological Diversity, Ecosystems and The Human Scale. Ecological Applications, 1018-1024.

22. FREIRE-GUEVARA, E. (2001). Baños en el tiempo y el espacio. Quito: Casa de la Cultura Ecuatoriana. 
23. FRIBORG, O., BARLAUG, D., MASRTINUSSEN, M., ROSENVINGE, J., \& HJEMDAL, O. (2005). Resilience in relation to personality and intelligence. International Journal of Methods in Psychiatric Research, 29-42.

24. GALL, M. (2013). From social vulnerability to resilience: measuring progress toward disaster risk reduction. Bonn: United Nations University.

25. García, R. (2006). Sistemas Complejos. Conceptos, método y fundamentación epistemológica de la investigación interdisciplinaria. Barcelona: Gedisa S.A.

26. Gobierno Autónomo Descentralizado del cantón Baños de Agua Santa . (2015). Diagnóstico del cantón Baños de Agua Santa (2014-2019). Baños: GAD Baños.

27. Godschalk, D. (2003). Urban Hazard Mitigation: Creating Resilient Cities. Natural Hazars Review, 136-143.

28. GRABHER, G., \& STARK, D. (1997). Organizing Diversity : Evolutionary Theory, Network Analysis and Postsocialism; Stark, David; . Regional Studies, 533-544.

29. GUNDERSON, L. (2000). Ecological Resilience - In Theory and Application. Annual Review Of Ecology and Systematics, 425-439.

30. GUNDERSON, L., \& HOLLING, C. S. (2002). Panarchy: Understanding Transformations in Human and Natural System. Washington DC: Island.

31. HEYLIGHEN, F. (2001). The science of self-organization and adaptivity. The Encyclopedia of Life Support Systems, 253-280.

32. HOLLAND, J. H. (1995). Hidden Order. Haw adaptation builds complexity. USA: Helix Books.

33. HOLLAND, J. H. (2006). Studying Complex Adapatative Systems. Jrl Syst Sci \& Complexity, 1-8.

34. HOLLING, C. S., \& GUNDERSON, L. H. (2002). Resilience and Adaptative Cycles. En L. H. Gunderson, \& C. S. Holling, Panarchy, Understanding Transformation in Human and Natural Systems. Washington: Island.

35. HOLLING, C. S., GUNDERSON, L. H., \& LUDWING, D. (2002). In Quest of a Theory of Adaptative Change. En L. H. Gunderson, \& C. S. Holling, Panarchy. Understanding Transformations in Human and Natural Systems. Washington: Island Press.

36. IFEJIKA, C., WIESMANN, U., \& RIST, S. (2014). An indicator framework for assessing livelihood resilience in the context of social-ecological dynamics. Global Environmental Change, 109119.

37. INEC. (2010). Censo Nacional de Población y Vivienda . Quito: Instituo Nacional de Estadísticas y Censos INEC.

38. INEC. (2010). Censo Nacional Económico. Quito: Instituto Ecuatoriano de Estadísticas y Censos INEC.

39. INEC. (2013). Directorio empresarial . Quito: INEC.

40. ISLAM, M., SWAPAN, M., \& HAQUE, S. (2013). Disaster risk index: How far should it take account of local attributes? International Journal of Disaster Risk Reduction, 76-87.

41. JOERIN, J., SHAW, R., TAKEUCHI, Y., \& KRISHNAMURTHY, R. (2012). Assessing community resilience to climate-related disaster in Chennai, India. International Journal of Disaster Risk Reduction, 44-54. 
42. LANE, L. R., TOBIN, G. A., \& WHITEFORD, L. M. (2003). Volcanic hazard or economic destitution: hard choices in Banños, Ecuador. Environmental Hazards, 23-34.

43. LASIO, V., CAICEDO, G., ORDEÑANA, \& VILLA, R. (2014). Global Entrepreneurship Monitor. Guayaquil: ESPAE- ESPOL.

44. MAILLAT, D. (1995). Desarrollo teritorial, milieu y política regional . En A. Vásquez, \& G. Garofoli, Desarrollo económico local en Europa (págs. 37-51). Madrid: Colegio de Economistas.

45. MARTIN, R., \& SUNLEY, P. (14 de octubre de 2013). On the Notion of Regional Economic Resilience : Conceptualisation and Explanation. Working paper about Evolutionary Geography. Utrecht University. Urban \& Regional research centre Utrech.

46. MORÍN, E. (1981). El Método: La Naturaleza de la Naturaleza. Madrid: Cátedra.

47. ORENCIO, P. M., \& FUJII, M. (2013). A localized disaster-resilience index to assess coastal communities based on an analytic hierarchy process (AHP). International Journal of Disaster Risk Reduction, 62-75.

48. ÖSTH, J., REGGIANI, A., \& GALIAZZO, G. (2015). Spatial economic resilience and accessibility: A join perspective. Computers, Environment and Urban Systems, 148-159.

49. PASOL, B. (2014). ¿Hacia una "nueva época" en los estudios de momoria social? Revista Mexicana de Ciencias Políticas y Sociales, 291-316.

50. PETERSON, G., ALLEN, C. R., \& HOLLING, C. S. (1998). Ecological Resilience, Biodiversity, and Scale Ecological Resilience, Biodiversity, and Scale. Ecosystems, 6-18.

51. PROAG, V. (2014). The concept of vulnerability and resilience. Procedia Economics and Finance, 369-376.

52. RODIMA-TAYLOR, D., \& OLWIG, M. C. (2012). Adaptation as innovation, innovation as adaptation: An institutional approach to climate change. Applied Geography, 107-111.

53. SCHNEIDER, B. (2007). Resiliencia: Cómo construir empresas exitosas en contextos de inestabilidad. Bogotá: Norma.

54. SCHUMPETER, J. A. (1942). Creative Destruction. Capitalism, Socialism and Democracy, 82-85.

55. SHERRIEB, K., NORRIS, F., \& GALEA, S. (2010). Measuring Capacities for Community Resilience. Social indicators research, 227-247.

56. SINGH-PETERSON, L., SALMON, P., GOODE, N., \& GALLINA, J. (2014). Translation and evaluation of the Baseline Resilience Indicators for Communities on the Sunshine Coast, Queensland Australia. International Journal of Disaster Risk Reduction, 116-126.

57. SMIT, B., \& WANDEL, J. (2006). Adaptation, adaptive capacity and vulnerability. Global enviromental Change, 282-292.

58. STARK, D. (2014). On Resilience. Social Sciences, 60-70.

59. TIERNEY, K., \& BRUNEAU, M. (2007). Conceptualizing and Measuring Resilience. A key to disaster loss reduction. TR News, 14-18.

60. TOBIN, G., \& WHITERFORD, L. (2002). Community resilience and volcano hazard: the eruption of Tungurahua and evacuation of the faldas in Ecuador. Disasters, 28-48.

61. UNISDR, T. U. (9 de septiembre de 2015). What's new. Obtenido de http://eird.org/news-oldversion.htm 
62. WALKER, B., \& SALT, D. (2012). Resilience Practice. Building Capacity to Absorb Disturbance and Maintain Function. Washington DC: Island Press.

63. WALKER, B., HOLLING, C. S., CARPENTER, S., \& KINZIG, A. (2004). Resilience, Adaptability and Transformability in Social - ecological Systems. Ecology and Society.

64. WHITEFORD, L., TOBIN, G., VINDROLA-PADROS, C., \& LASPINA, C. (2013). We have to think about the children: parenting responses in chronic natural disasters. Emergency Management, 59-75.

65. WILSON, E. (1992). The diversity of life. Cambridge, Masaachusetts, USA: Belknap.

66. WINDLE, G., BENNETT, K., \& NOYES, J. (2011). A methodological review of resilience measurement scales. Health and quality of life outcomes, www.hqlo.com/content/9/1/8.

67. ZOBEL, C., \& KHANSA, L. (2014). Characterizing multi-event disaster resilience . Computers and Operations Research, 83-94. 